\title{
Imaging Early Steps of Sindbis Virus Infection by Total Internal Reflection Fluorescence Microscopy
}

\author{
Youling Gu, Yuanzheng Yang, and Yuechueng Liu \\ Department of Pathology, University of Oklahoma Health Sciences Center, P.O. Box 26901, Oklahoma City, Ok 73190, USA \\ Correspondence should be addressed to Yuechueng Liu, yuechueng-liu@ouhsc.edu
}

Received 11 July 2011; Revised 7 September 2011; Accepted 8 September 2011

Academic Editor: Amiya K. Banerjee

Copyright () 2011 Youling Gu et al. This is an open access article distributed under the Creative Commons Attribution License, which permits unrestricted use, distribution, and reproduction in any medium, provided the original work is properly cited.

Sindbis virus (SINV) is an alphavirus that has a broad host range and has been widely used as a vector for recombinant gene transduction, DNA-based vaccine production, and oncolytic cancer therapy. The mechanism of SINV entry into host cells has yet to be fully understood. In this paper, we used single virus tracking under total internal reflection fluorescence microscopy (TIRFM) to investigate SINV attachment to cell surface. Biotinylated viral particles were labeled with quantum dots, which retained viral viability and infectivity. By time-lapse imaging, we showed that the SINV exhibited a heterogeneous dynamics on the surface of the host cells. Analysis of SINV motility demonstrated a two-step attachment reaction. Moreover, dual color TIRFM of GFP-Rab5 and SINV suggested that the virus was targeted to the early endosomes after endocytosis. These findings demonstrate the utility of quantum dot labeling in studying the early steps and behavior of SINV infection.

\section{Introduction}

Sindbis virus (SINV) is an alpha virus and a member of the Toga virus family that also includes Semliki Forest and Ross River viruses [1]. It was first isolated in 1952 from mosquitoes found in the Sindbis area in Egypt [2]. The decoded SINV genome revealed a single-strand RNA of $11.7 \mathrm{~kb}$ in size [3]. Upon cell entry, the viral RNA strand serves as a messenger RNA to translate four nonstructure genes that are essential for viral replication. In late stages of infection, structural genes are translated and synthesized from a subgenomic RNA. The resulting viral particles containing nucleocapsids of genomic RNA and capsid proteins, packaged with envelope proteins E1/E2, are formed and budded off as mature virus $[4,5]$. The receptors that are involved in SINV entry into host cells remain poorly characterized. One of such receptors is the $67 \mathrm{kD}$ high-affinity laminin receptor (LR), which has been shown to mediate SINV infection of BHK cells $[6,7]$. In addition, it has been reported that heparan sulfate, a major cell surface component, is directly involved in SINV infection of cultured cells $[6,8]$. Little is known regarding viral attachment and movement on host cell surfaces during early stages of SINV infection.
SINV has long been used as an experimental model for studying encephalitis because it causes encephalomyelitis in neonatal mice [9]. Moreover, since SINV has a broad host range and can deliver efficient gene expression, vectors based on the virus have been developed for vaccine production and gene therapy purposes $[10,11]$. In cancer therapy, SINV has been tested as a potential oncolytic reagent. It has been shown that a SINV strain (Toto1101) derived from the wildtype SINV was effective targeting and killing tumor cells of ovarian, colon, prostate, and liver cancer patients, leading to tumor regression in animal models $[12,13]$.

In recent years, quantum-dot- (Qdot-) based fluorescence labeling has become increasingly employed for imaging cellular events including single-molecule tracking and livecell imaging [14-16]. Qdots are semiconductor nanocrystals with a broad excitation and emission spectra. One advantage of Qdot is its resistance to photobleaching, allowing prolonged exposure to excitation light. In the current study, we labeled SINV with biotin and conjugated the virus with Qdot 605. Using single-virus tracking, we were able to dissect the behavior and dynamics of individual SINV during the initial stages of infection, which showed a two-step, receptormediated cell attachment process. 


\section{Materials and Methods}

2.1. Cell Culture and SINV Preparation. BHK-21 cells were cultured in MEM supplemented with 10\% FBS and antibiotics. The cells were maintained in a humidified $37^{\circ} \mathrm{C}$ incubator with $5 \% \mathrm{CO}_{2}$. For the construction of SINV-GFP expressing the green fluorescent protein (GFP), GFP cDNA (from pS65T-C1, Clonetech) was first cloned into a shuttle vector $\mathrm{pH} 2 \mathrm{~J} 1 \mathrm{Y}$ which was modified from $\mathrm{pH} 3^{\prime} 2 \mathrm{~J} 1$ (kindly provided by Dr. Guangpu Li, University of Oklahoma, Ok, USA). The plasmid $\mathrm{pH} 2 \mathrm{~J} 1 \mathrm{Y}$ contained a linker with additional multiple cloning sites, Kpn I, Sal I, EcoR V, Hind III, and Nhe I, which was inserted between the Xba $\mathrm{I}$ and Bam $\mathrm{H} 1$ sites of $\mathrm{pH} 3^{\prime} 2 \mathrm{~J} 1$. The cDNA encoding the GFP was excised by Nhe I and Bam $\mathrm{H} 1$ and ligated into pH2J1Y at same sites. An Apa I-Xho I fragment of pH2J1Y harboring the GFP cDNA was subcloned into the SINV vector pToto/3'2J. To construct SINV-C'-YFP, in which the 62-106 amino acid sequence of the capsid protein was replaced with EYFP, an Sph I and an Mlu I sites flanking the replaced capsid sequence were introduced into pToto/3'2J by PCR mutagenesis. EYFP with Sph I and Mlu I cloning sites was amplified by PCR using pEYFP-C1 as a template. Recombinant SINV was produced by transfection of BHK21 cells using capped RNAs derived from sp6 RNA polymerase transcription of Xho I-linearized plasmid templates. Viral stocks were obtained by harvesting the culture supernatants $30 \mathrm{hr}$ after infection and aliquots were stored at $-80^{\circ} \mathrm{C}$ until use. Viral titers were determined by plaque assay on BHK21 monolayers using crystal violet staining.

2.2. Biotinylation of SINV. SINV-GFP was collected in serum free MEM diluted with saline $(1: 1)$ at $\sim 4 \times 10^{8} \mathrm{pfu} / \mathrm{mL}$ and was labeled with biotin at room temperature or on ice. Briefly, the virus was mixed with $10 \mathrm{mM}$ stock NHSPEG4-Biotin (29 $\AA$ spacer arm, Pierce) in PBS ( $\mathrm{pH} 8.0$ ) at a final concentration of $300 \mu \mathrm{M}$ for $30 \mathrm{~min}$ at room temperature. The reaction was terminated by adding glycine to $0.1 \mathrm{M}$ and incubating for $15 \mathrm{~min}$ at room temperature. For biotinylation on ice, SINV was incubated with NHS-PEG4Biotin as described above for 90 minutes on ice. The reaction was terminated by addition of glycine and further incubation at room temperature for 20 minutes. We did not notice any significant differences in the efficiency of biotinylation and the infectivity of the resulting biotinylated SINV produced under the two labeling conditions. For western blot analysis of the labeled SINV, aliquots of SINV were heated at $100^{\circ} \mathrm{C}$ for $3 \mathrm{~min}$ in sample buffer containing 1\% SDS, $10 \mathrm{mM}$ EDTA, $10 \mathrm{mM}$ DTT, $15 \%$ glycerol, $20 \mathrm{mM}$ Tris- $\mathrm{HCl}, \mathrm{pH}$ 6.8 , and $0.01 \%$ bromophenol blue. The SINV proteins were separated by SDS-PAGE and transferred onto nitrocellulose membrane. An alkaline phosphatase-conjugated streptavidin ( $1: 5,000$, Sigma) was used to visualize biotinylated SINV envelope proteins.

2.3. SINV Infection, Fluorescence Microscopy, and Image Analysis. Biotinylated SINV was used to infect BHK-21 cells at MOI (multiplicity of infection) of 5-20. The virus was incubated with the cells at $4^{\circ} \mathrm{C}$ for 1 hour. After wash- ing with PBS to remove unbound virus, the cells were further incubated with streptavidin-conjugated Qdot 605 (Invitrogen) for 60 minutes and washed 3 times with cold PBS. The cells were fixed with 3.7\% formaldhyde in PBS for 30 minutes at room temperature. Fluorescent images were acquired and digitized for analysis. For live cell experiments, the infection was performed on ice or at $4^{\circ} \mathrm{C}$ for 1 hour before shifting to a $35^{\circ} \mathrm{C}$ chamber for microscopy.

For TIRFM, we used an Olympus IX71 equipped with a $60 \mathrm{X}$ (n.a. $=1.45$ ) $\mathrm{TIRF}$ objective heated to $37^{\circ} \mathrm{C}$. Cells were cultured in a home-made glass bottom dish and maintained at $35^{\circ} \mathrm{C}$ in an environmental control chamber (Olympus, USA) supplemented with $5 \% \mathrm{CO}_{2}$. TIRF illumination was achieved using a $20 \mathrm{~mW} 488 \mathrm{~nm}$ laser source for excitation and a long-pass dichroic beam splitter (500 nm). GFP/YFP was viewed with an emission filter of $525 / 20 \mathrm{~nm}$, and Qdot 605 was viewed with an emission filter of 600/40 nm. Emission filters were controlled with a Lambda 10 (Sutter Instrument, Novato, CA, USA) high-speed filter wheel. Images were captured with a CCD camera (Quantix 57, Photometrics, Tucson, Ariz, USA) air cooled to $-25^{\circ} \mathrm{C}$. The camera was controlled by IPlab 3.9.4 (Scanalytics, Fairfax, Va, USA) and analyzed with the IPlab software. For expression of GFP-Rab5, BHK-21 cells were transfected using Lipofectamine 2000 (Invitrogen) and pGFP-Rab5 (Dr. Guangpu Li, University of Oklahoma Health Sciences Center). The cells expressing GFP-Rab5 were imaged under TIRFM 24-48 hours after transfection.

\section{Results}

3.1. Labeling SINV with Qdot 605. SINV was first conjugated to biotin linked through a $29 \AA \mathrm{PEG}$ arm. In order to achieve a near $100 \%$ labeling of SINV, an excessive amount of NHS-biotin at $300 \mu \mathrm{M}$ was used in the reaction. This was necessary for ensuring that the observed infection was indeed by the biotinylated SINV, not by the native and un-modified viruses. The conjugation reaction, however, reduced the infective titer of the virus from $4 \times 10^{8}$ to $10^{6}-$ $10^{7} \mathrm{pfu} / \mathrm{mL}$. Such a reduction was mostly due to the excessive chemical modification by NHS-biotin since viral titer was less affected when the concentration of NHS-biotin was reduced (data not shown). Western blot analysis showed that both the E1/E2 envelope proteins were labeled (Figure 1(a)). Moreover, the excessive crosslinking caused some E1/E2 proteins shifting to slightly higher apparent molecular weight (Figure 1(a)). When the biotin-SINV was preincubated with Qdot 605, a medium-sized quantum dot of 5-12 nm diameter, the virus-Qdot conjugate was still active infecting BHK21 cells with a small reduction in infection activities $(<2$ fold). However, if we preincubated the cells with SINV for 1 hour at $4^{\circ} \mathrm{C}$ and then added Qdot, the reduction in infectivity became minimum. Specific binding to host cells by Qdotlabeled SINV was evident under fluorescence microscopy (Figure 1(b)). To determine if the Qdot-SINV was able to enter cells and carry out viral replication, we followed the expression of recombinant GFP encoded by the viral vector. BHK-21 cells were scored for GFP and Qdot 605 fluorescence 14 hours after infection, following the completion of first 


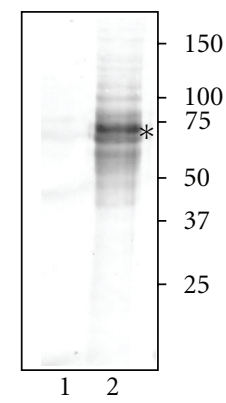

(a)
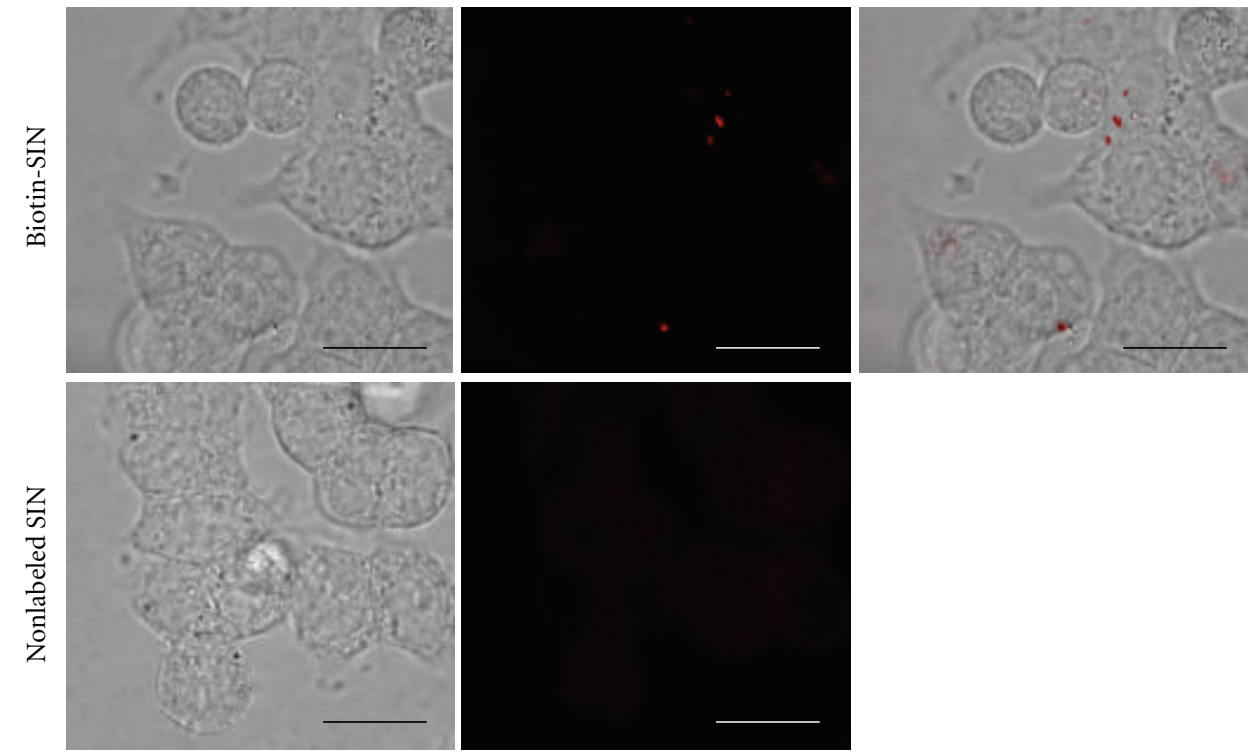

(b)
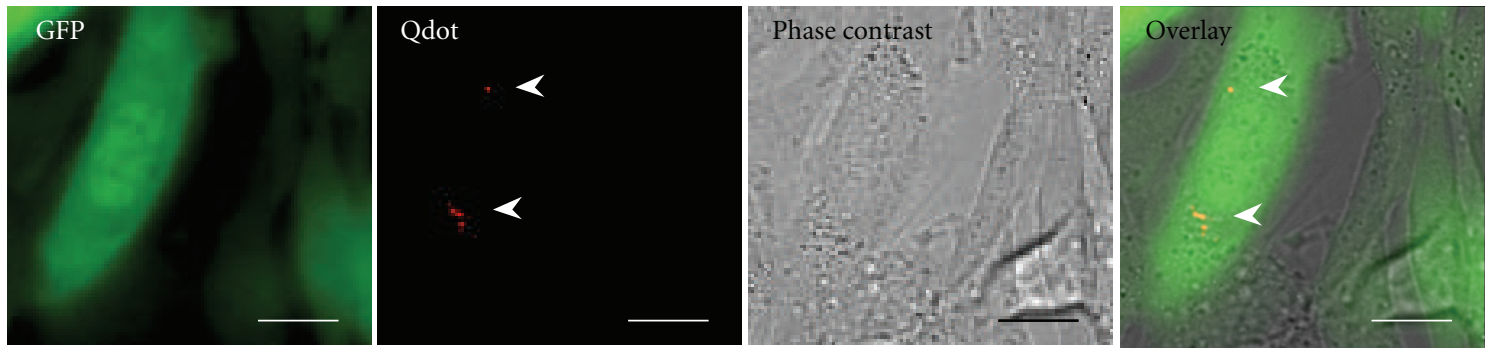

(c)

FIgURE 1: Biotinylation and Qdot labeling of SINV. SINV was labeled with NHS PEG4-biotin as described in Section 2. (a) Western blot analysis of biotinylated SINV using alkaline-phosphatase-conjugated streptavidin showing efficient labeling of the E1/E2 envelope proteins (indicated by ${ }^{*}$ ). Lanes 1: control unlabeled SINV and 2: biotinylated SINV. Molecular weight markers $(\mathrm{kD})$ are labeled. (b) Biotinylated SINV labeled with Qdot 605 specifically bound target cells. Arrows indicate Qdot-labeled SINV. Bar $=25 \mu \mathrm{m}$. (c) Biotinylated SINV labeled with Qdot 605 was active infecting target cells and expressing GFP. Images were taken 14 hours after infection. Arrowheads indicate Qdot signals. Bar $=25 \mu \mathrm{m}$.

round of infection. Examination of randomly selected cells revealed that $>70 \%$ cells expressing GFP $(n=500)$ were also positive for Qdot 605 (Figure 1(c)), which suggested that the Qdot-labeled SINV was able to enter the cells and continue to replicate and express viral genome.

3.2. Dynamics of Qdot-Labeled SINV on BHK Cell Surface. To study the dynamics of SINV on host cell plasma membranes, we used time-lapsed microscopy to record the movement of the virus in real time under TIRF. In order to synchronize viral attachment and to inhibit viral entry, we performed the incubation reactions at $4^{\circ} \mathrm{C}$ or on ice. This would presumably arrest SINV at surface attachment stage. The cell culture was then shifted to a $35^{\circ} \mathrm{C}$ environmental control chamber for microscopy. We first recorded the movement of the SINV-C'-YFP, which had a 44-amino-acid domain 

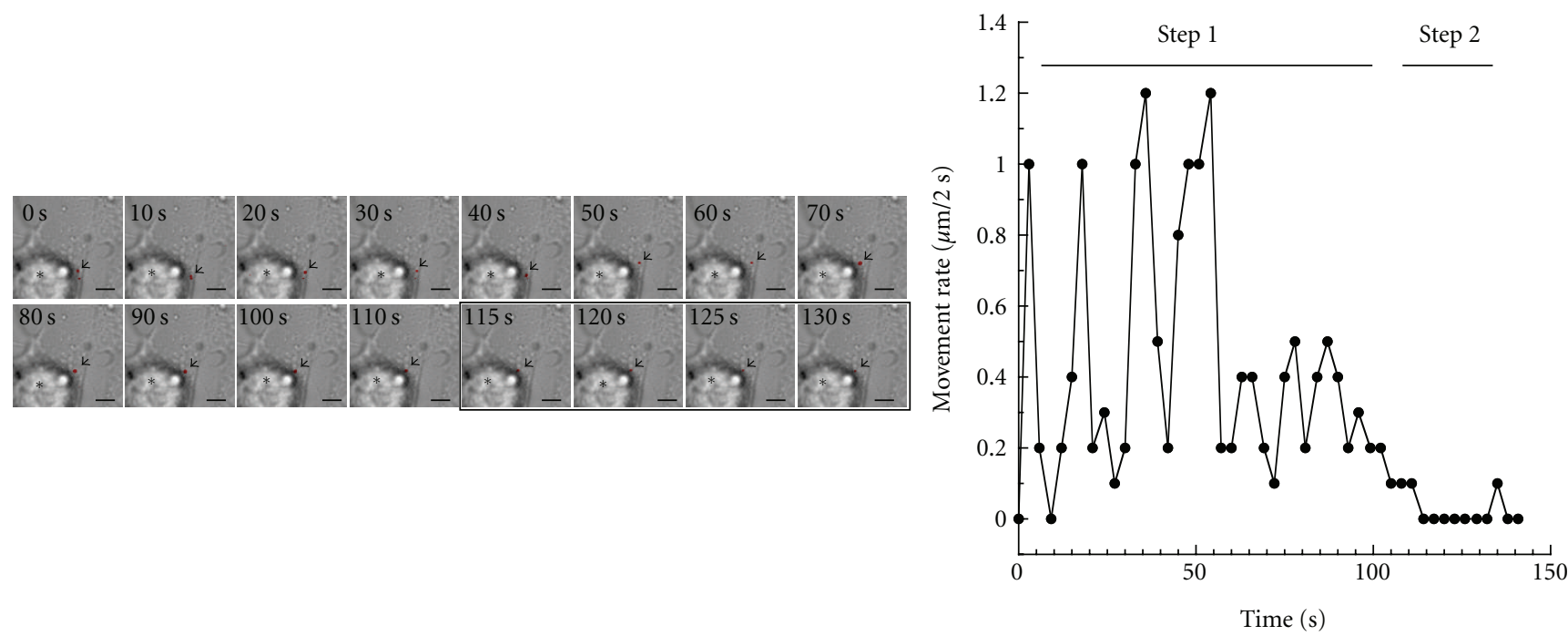

(a)
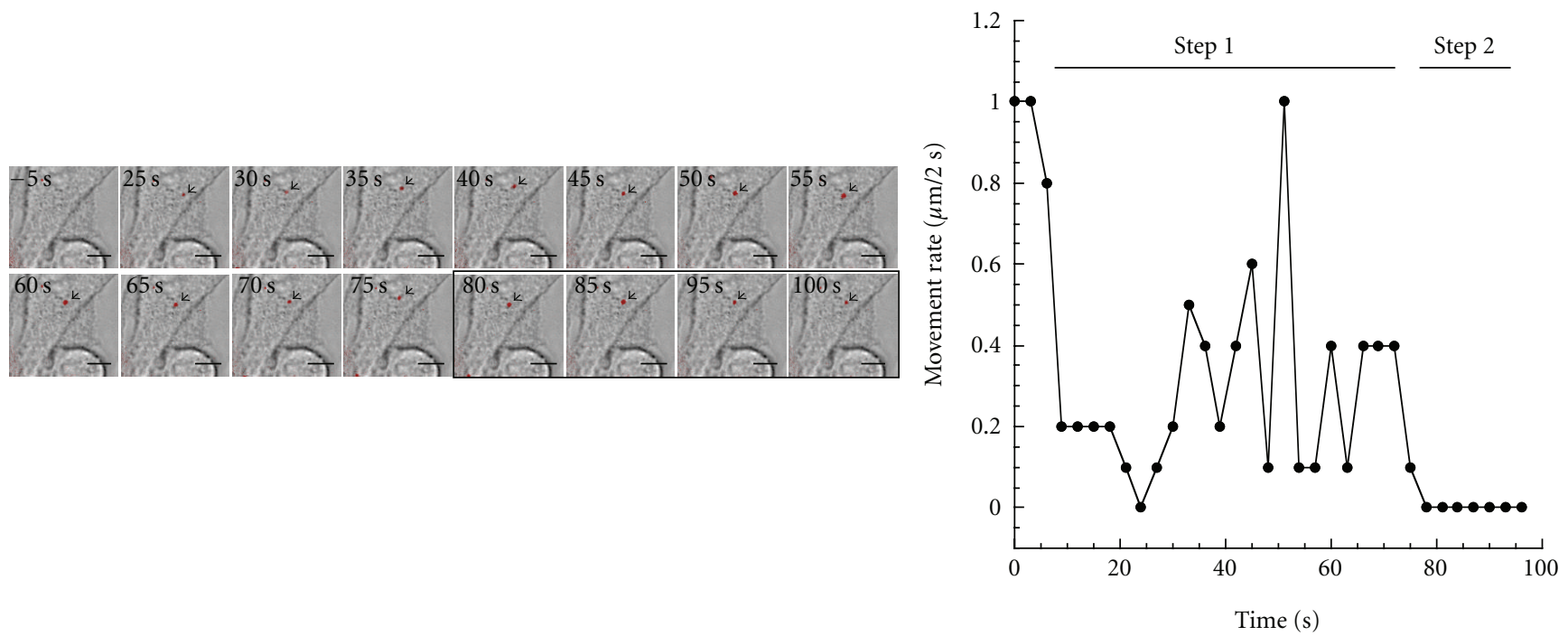

(b)

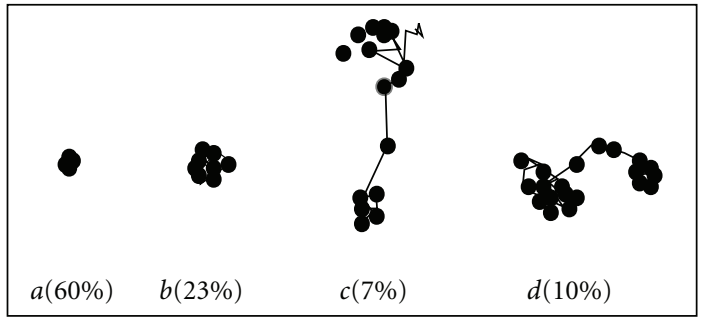

(c)

FIGURE 2: Single-particle tracking of a SINV virus binding to cell surface. Pseudocolor images showing that SINV attachment involved a two-step binding process. (a) A SINV-C'-YFP (arrow) was tracked under TIRF mode for 130 seconds. Its movement rate was measured manually frame-by-frame. Boxed images indicate no measurable movement by the virus. (b) Tracking of a SINV labeled with Qdot 605. (c) Four types of representative trajectories of Qdot-labeled SINV movement in BHK plasma membranes. $a$ : immobile; $b$ : mobile but confined in small areas $(<0.5 \mu \mathrm{m}$ diameter $)$; : mobile through long distances $(>1 \mu \mathrm{m}) ; d$ : mobile through medium distances $(0.5-1 \mu \mathrm{m})$. Scale bar $=$ $10 \mu \mathrm{m}$. 

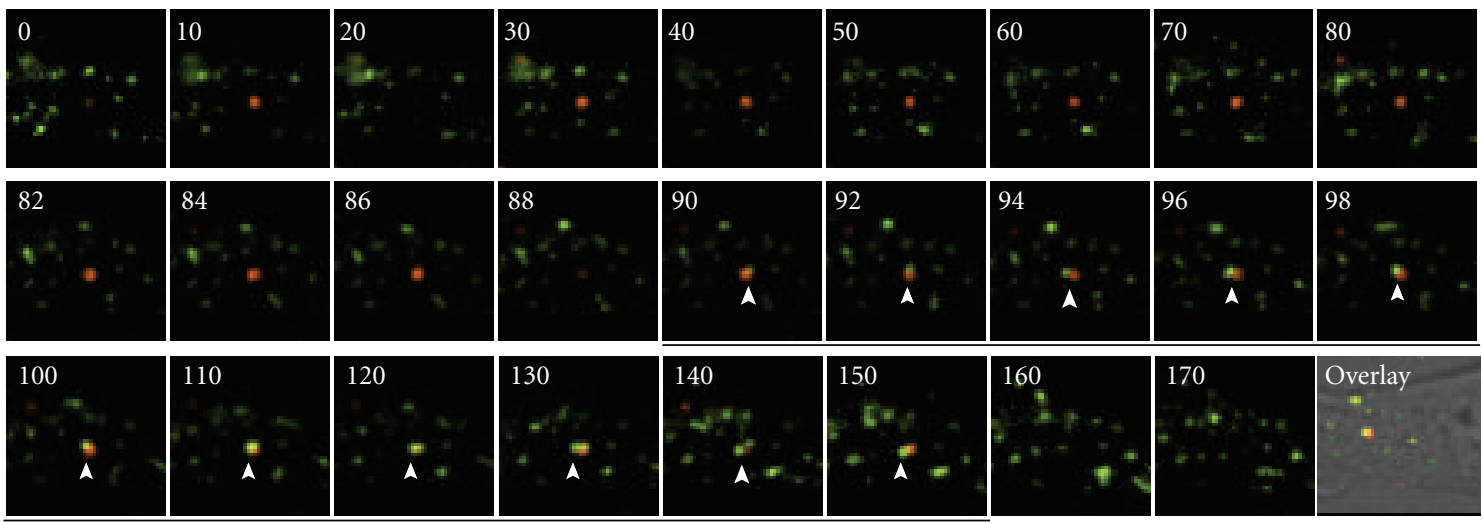

FIGURE 3: Dual color TIRFM of SINV and Rab-5 after endocytosis. A SINV virus after entering the cell was tracked (red). After approximately 90 seconds, it was associated with GFP-Rab5 (green). The association lasted $~ 60$ second before the GFP-Rab5 dissociated and the SINV moved deeper into the cell. Time in seconds is labeled. Arrowheads indicate the association of Rab5. The last image shows an overlay composite picture of the cell.

of the capsid protein replaced with EYFP. This virus was shown to retain the same infectivity as the wild-type virus generated from pToto/3'2J (data not shown). As shown in Figure 2(a), the virus moved rapidly in a random fashion at rates ranging from 0 to $600 \mathrm{~nm} /$ second. We were able to identify two types of virus-receptor association based on the rate of movement. One was brief and transient, which was exemplified by rapid movement of the virus. The other type of virus-receptor interaction appeared to be firm attachment, accompanied by the immobilization of the virus (Figure 2(a)). When we tracked SINV labeled with Qdot 605, the virus also exhibited the two types of cell association as the SINV-C'-YFP. The movement of attached Qdot-SINV appeared similar to that of SINV-C'-YFP, with a max rate of $\sim 500 \mathrm{~nm} /$ second (Figure 2(b)). The SINV moved rapidly with a random trajectory. There were frequent associations and disassociations between the virus and cell surface. Most interactions appeared brief and transient. Full viral attachment became evident when the virus was firmly bound to the cell surface and became nearly immobile (Figure 2(b)). This finding suggested that Qdot did not significantly impact on the property of virus-receptor interactions. To investigate further into the behavior of Qdot-labeled SINV during cell surface attachment, we collected images from multiple cells $(n=200)$ and recorded 100 frames at 2 sec per frame rate for each cell. As illustrated in Figure 2(c), we observed 4 typical movements of SINV on cell surface. Nearly $60 \%$ of the viral particles were virtually immobile, indicating that they were likely bound to receptors nonspecifically or they might be functionally impaired from Qdot labeling. The other $40 \%$ of the SINV particles showed a heterogeneous lateral motility along the membranes. While some $(23 \%)$ were confined in a small area $\left(<0.25 \mu \mathrm{m}^{2}\right)$, others $(17 \%)$ were moving beyond several $\mu \mathrm{m}$ in distance. The rate of SINV movement was also heterogeneous, ranging from 0 to $500 \mathrm{~nm}$ a second (Figure 2). It appeared that the virus often had preference for certain sites and would move in the vicinity. Moreover, movement would dramatically slow down once the virus settled on a particular site (Figure 2). These results suggested a two-step attachment process for the motile SINV. A first step involved a highly mobile receptor, and a second step involved the immobilization of the receptor/virus, perhaps prior to viral internalization (Figure 2).

3.3. Tracking SINV after Cell Entry. SINV has been shown to undergo clathrin-dependent endocytosis after receptor binding [17]. However, the fate of the virus following endocytic reaction remains to be fully delineated. By tracking single SINV particles labeled with Qdot 605, we were able to determine that SINV was transported via Rab5containing early endosomes. BHK cells expressing GFP-Rab5 were infected with Qdot-labeled SINV, and the virus was tracked at $35^{\circ} \mathrm{C}$ under TIRF. By taking the advantage of the superior optical sectioning capability of TIRFM, we were able to identify an internalized SINV based on its presence in the same optical plane with Rab5 under TIRF. As shown in Figure 3, SINV was targeted to Rab5-containing early endosomes after internalization. The co-localization of SINV with Rab5 lasted from 15 to 60 seconds, which indicated a rapid maturation of the early endosomes to late endosomes. Interestingly, about $40 \%$ of the SINV did not colocalize with GFP-Rab5 (data not shown).

\section{Discussion}

Quantum dots have been used in a wide range of applications including visualization of hepatitis C virus infection in human liver [18], tracking single SV40 virus [19], and imaging HER2 on tumor cells in real time [20]. Using Qdotbased single-virus tracking, we were able to begin dissecting the early events involving SINV infection. We have shown that, during a productive infection, SINV employed a twostep binding reaction to achieve high-affinity binding with its receptors. The first binding reaction resulted in a bound virus that was highly mobile and dynamic on the host cell surface. This binding often did not lead to viral internalization and could last from seconds to several minutes. The second step was high-affinity binding and attachment, which was accompanied by drastically reduced viral mobility. 
The immobilization of the virus might be a necessary step before viral endocytosis. After internalization, Qdot-SINV appeared to be transported via early endosomes containing Rab5 (Figure 3), which was consistent with previous reports on Semliki Forest virus [21]. However, approximately $40 \%$ of the Qdot fluorescence did not colocalize with Rab5-GFP (data not shown). One possibility for such a significant amount of Qdot-SINV without GFP-Rab5 co-localization was that they involved Rab5-negative endosome domains, similar to those reported for Semliki Forest virus [21]. Alternatively, due to Qdot's highly stable and strong fluorescence, it was difficult to record low levels of Rab5-GFP fluorescence, especially after long exposure ( $>2$ minutes). Thus, the association of the Qdot-SINV with Rab5-negative compartment remains to be further investigated.

One advantage for quantum dots is that they are highly resistant to photobleaching. This allows prolonged excitation of the sample of interest, which in some instance requires minutes to hours of observation. In our experience using a $20 \mathrm{~mW} 488 \mathrm{~nm}$ laser, the Qdot 605 retained nearly $100 \%$ fluorescence intensity after 3 minutes of continuous illumination under TIR. In comparison, the EYFP showed a dramatic reduction of fluorescence emission after only 30 seconds of excitation and lost $>90 \%$ of the intensity after 3 minutes. Consequently, tracking the SINV-C'-YFP was limited to $<2$ minutes, making it difficult to study the virus after internalization. With Qdot-labeled SINV, we were able to follow the virus for up to an hour, which greatly expanded data collection on viral infection process. Another benefit of quantum dots is that they have broad excitation spectra, which allows simultaneous excitation of multiple fluorophores. As shown in Figure 3, we were able to perform dual TIRF imaging using a single excitation source.

The limitation of quantum dots is their relatively large sizes, which range from 10 to $20 \mathrm{~nm}$. When conjugated to biomolecules such as the E1/E2 envelope proteins of SINV, they could potentially interfere with the normal functions of the proteins. The greatest loss of viral infectivity was due to the initial biotinylation reaction. It appeared that some viruses were rendered inactive due to excessive biotinylation. Interestingly, binding of streptavidin-Qdot 605 had less adverse effect on SINV infection, which was further improved by adding the Qdot after SINV attachment to the cell surface. The relatively benign hindering effect upon Qdot binding to SINV suggested that, at an average 5$12 \mathrm{~nm}$ diameter, Qdot 605 did not significantly affect the infectivity of SINV. We believe this is likely due to the fact that Qdot 605 bound with SINV mostly at an 1:1 ratio. Nevertheless, conditions for the conjugation process can be further optimized to minimize hindering, which will make quantum-dot-based tracking a more widely used tool for investigating molecular details of viral infection.

\section{Acknowledgments}

The authors thank Dr. Guangpu Li (University of Oklahoma Health Sciences Center) for providing the pToto/3'2J plasmid and pGFP-Rab5 vector. This work was supported by grants from the Oklahoma Center for the Advancement of Science and Technology (HR07-056) and Sarcoma Foundation of America.

\section{References}

[1] J. H. Strauss and E. G. Strauss, "The alphaviruses: gene expression, replication, and evolution," Microbiological Reviews, vol. 58, no. 3, pp. 491-562, 1994.

[2] R. M. Taylor, H. S. Hurlbut, T. H. Work, J. R. Kingston, and T. E. Frothingham, "Sindbis virus: a newly recognized arthropodtransmitted virus," The American Journal of Tropical Medicine and Hygiene, vol. 4, no. 5, pp. 844-862, 1955.

[3] E. G. Strauss, C. M. Rice, and J. H. Strauss, "Complete nucleotide sequence of the genomic RNA of Sindbis virus," Virology, vol. 133, no. 1, pp. 92-110, 1984.

[4] J. A. Lemm and C. M. Rice, "Roles of nonstructural polyproteins and cleavage products in regulating Sindbis virus RNA replication and transcription," Journal of Virology, vol. 67, no. 4, pp. 1916-1926, 1993.

[5] Y. Shirako and J. H. Strauss, "Regulation of Sindbis virus RNA replication: uncleaved $\mathrm{P} 123$ and nsP4 function in minusstrand RNA synthesis, whereas cleaved products from P123 are required for efficient plus-strand RNA synthesis," Journal of Virology, vol. 68, no. 3, pp. 1874-1885, 1994.

[6] W. B. Klimstra, K. D. Ryman, and R. E. Johnston, "Adaptation of Sindbis virus to BHK cells selects for use of heparan sulfate as an attachment receptor," Journal of Virology, vol. 72, no. 9, pp. 7357-7366, 1998.

[7] K. S. Wang, R. J. Kuhn, E. G. Strauss, S. Ou, and J. H. Strauss, "High-affinity laminin receptor is a receptor for Sindbis virus in mammalian cells," Journal of Virology, vol. 66, no. 8, pp. 4992-5001, 1992.

[8] A. P. Byrnes and D. E. Griffin, "Binding of Sindbis virus to cell surface heparan sulfate," Journal of Virology, vol. 72, no. 9, pp. 7349-7356, 1998.

[9] S. Lustig, A. C. Jackson, C. S. Hahn, D. E. Griffin, E. G. Strauss, and J. H. Strauss, "Molecular basis of Sindbis virus neurovirulence in mice," Journal of Virology, vol. 62, no. 7, pp. 2329-2336, 1988.

[10] S. C. Derrick, A. L. Yang, and S. L. Morris, "Vaccination with a Sindbis virus-based DNA vaccine expressing antigen 85B induces protective immunity against Mycobacterium tuberculosis," Infection and Immunity, vol. 73, no. 11, pp. 7727-7735, 2005.

[11] H. V. Huang, "Sindbis virus vectors for expression in animal cells," Current Opinion in Biotechnology, vol. 7, no. 5, pp. 531$535,1996$.

[12] C. Li, Y. Gu, D. Andrade, and Y. Liu, "Susceptibility of colorectal cancer cells to sindbis virus infection," Journal of Experimental Therapeutics and Oncology, vol. 8, no. 2, pp. 167$175,2009$.

[13] J. C. Tseng, B. Levin, A. Hurtado et al., "Systemic tumor targeting and killing by Sindbis viral vectors," Nature Biotechnology, vol. 22, no. 1, pp. 70-77, 2004.

[14] S. R. Nelson, M. Y. Ali, K. M. Trybus, and D. M. Warshaw, "Random walk of processive, quantum dot-labeled myosin Va molecules within the actin cortex of COS-7 cells," Biophysical Journal, vol. 97, no. 2, pp. 509-518, 2009.

[15] F. Pinaud, X. Michalet, L. A. Bentolila et al., "Advances in fluorescence imaging with quantum dot bio-probes," Biomaterials, vol. 27, no. 9, pp. 1679-1687, 2006.

[16] E. Saint-Michel, G. Giannone, D. Choquet, and O. Thoumine, "Neurexin/neuroligin interaction kinetics characterized by 
counting single cell-surface attached quantum dots," Biophysical Journal, vol. 97, no. 2, pp. 480-489, 2009.

[17] L. DeTulleo and T. Kirchhausen, "The clathrin endocytic pathway in viral infection," EMBO Journal, vol. 17, no. 16, pp. 4585-4593, 1998.

[18] Y. Liang, T. Shilagard, S. Y. Xiao et al., "Visualizing hepatitis $\mathrm{C}$ virus infections in human liver by two-photon microscopy," Gastroenterology, vol. 137, no. 4, pp. 1448-1458, 2009.

[19] P. Kukura, H. Ewers, C. Müller, A. Renn, A. Helenius, and V. Sandoghdar, "High-speed nanoscopic tracking of the position and orientation of a single virus," Nature Methods, vol. 6, no. 12, pp. 923-927, 2009.

[20] H. Tada, H. Higuchi, T. M. Wanatabe, and N. Ohuchi, "In vivo real-time tracking of single quantum dots conjugated with monoclonal anti-HER2 antibody in tumors of mice," Cancer Research, vol. 67, no. 3, pp. 1138-1144, 2007.

[21] A. Vonderheit and A. Helenius, "Rab7 associates with early endosomes to mediate sorting and transport of Semliki forest virus to late endosomes," PLoS Biology, vol. 3, no. 7, pp. 12251238, 2005. 

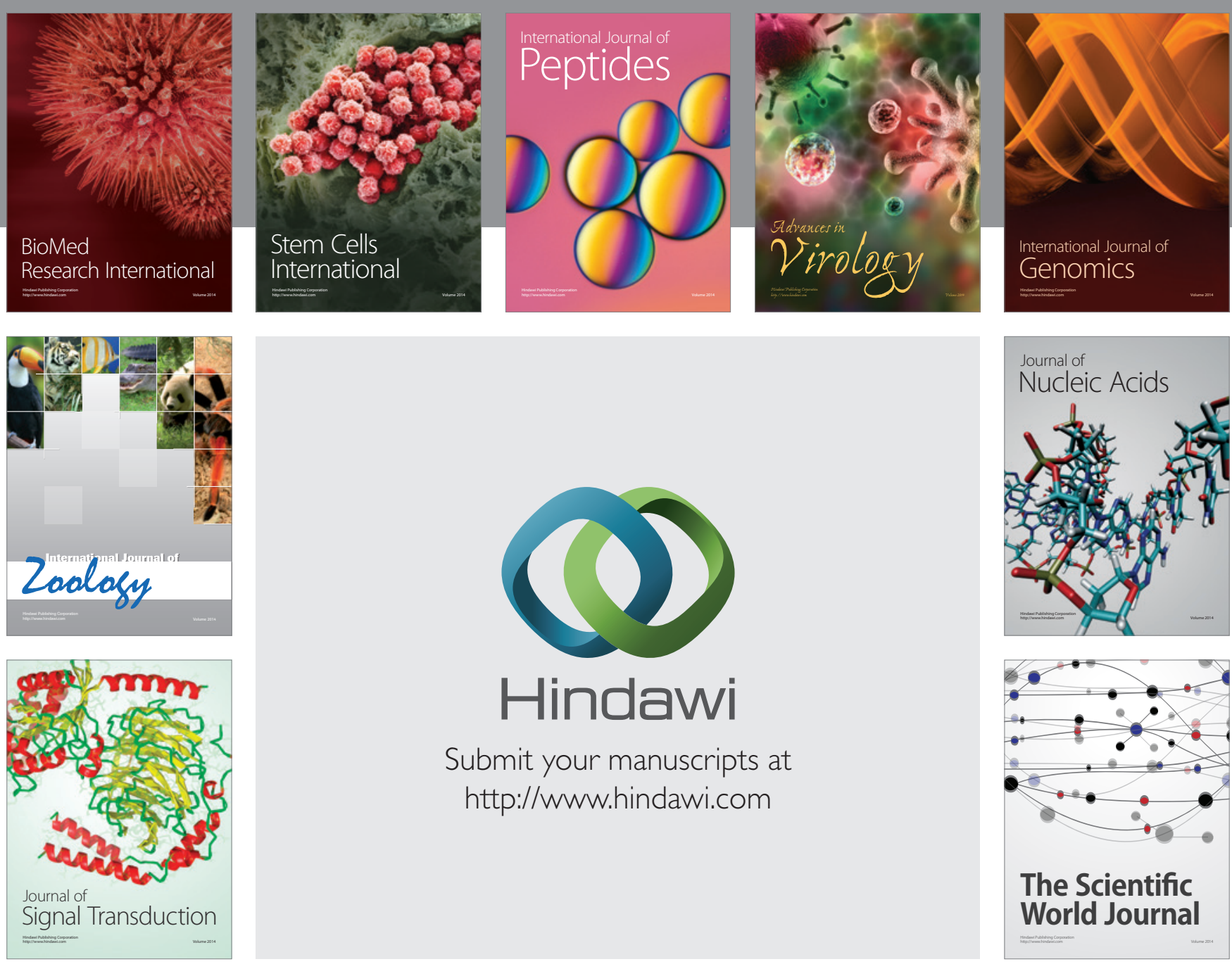

Submit your manuscripts at

http://www.hindawi.com
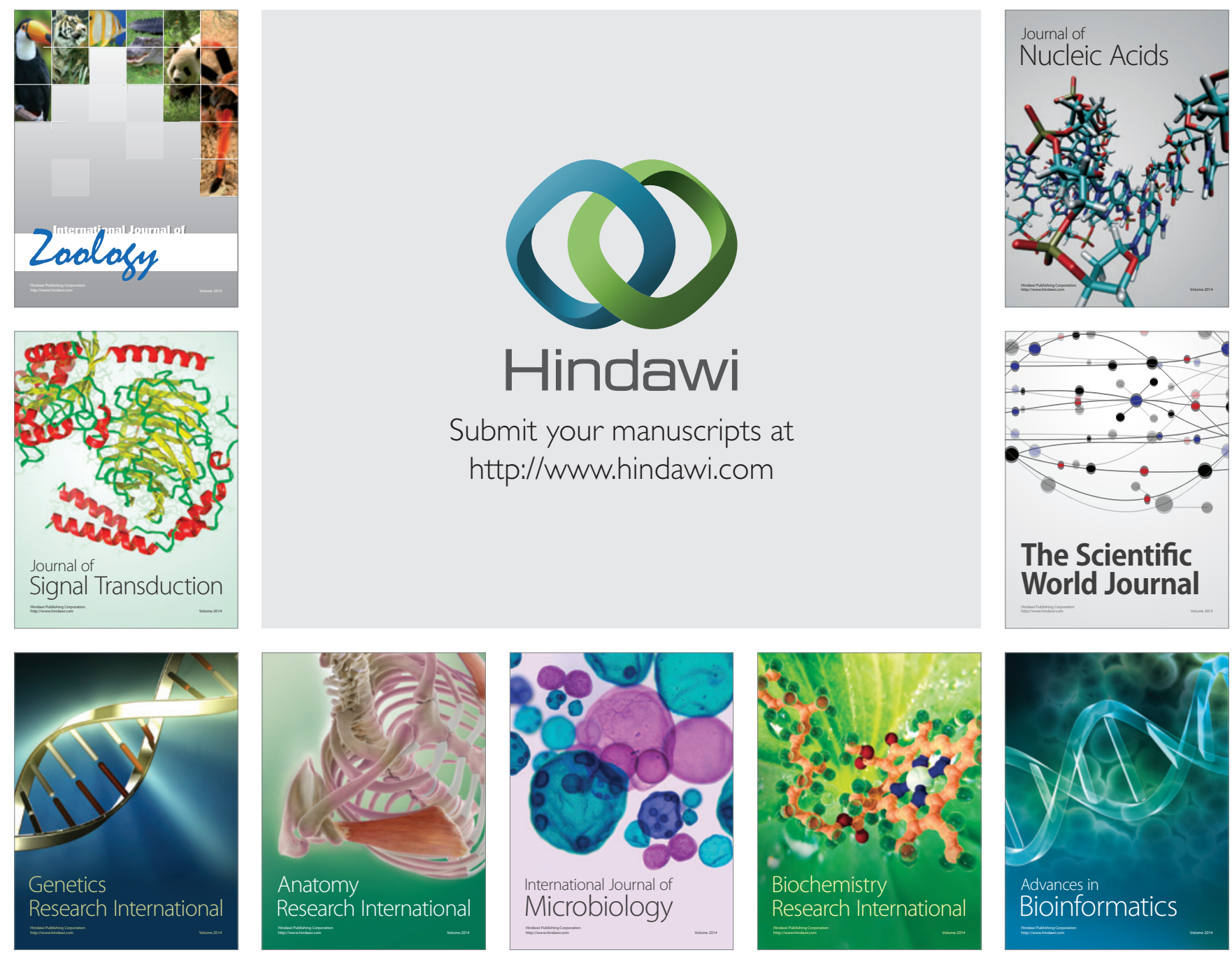

The Scientific World Journal
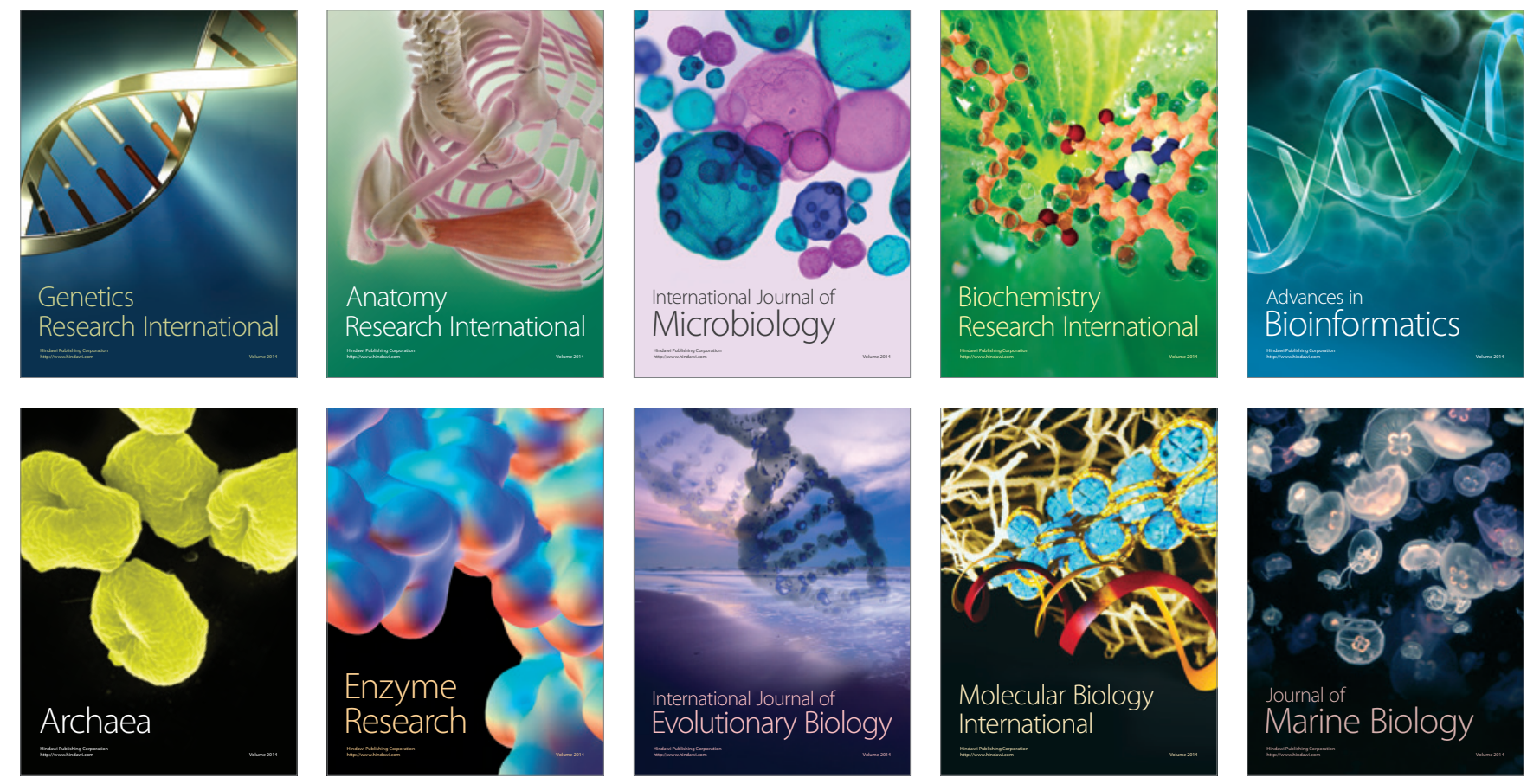